\title{
Research on Inferring ELECTRE-III's Parameters with Fuzzy information and A Case on Naval Gun Weapon System Integration
}

\author{
Shi Yan Sun ${ }^{1}$, Hui Min Zhu ${ }^{2}$ \\ ${ }^{1}$ Institute of Weaponry Engineering, Wuhan Navy University of Engineering, China \\ ${ }^{2}$ College of Electronics Engineering, Wuhan Naval University of Engineering, China
}

\begin{abstract}
Multiple attributes decision making (MADM) method is an important measure for system integration. Robustness analysis on MADM is a hotspot in these years which wins academe's great attention, and is supposed to be an effective way when countering imperfect information. Setting parameters in ELECTRE-III is a vital and difficult step. In this paper, a method of inferring ELECTRE-III' s parameters with fuzzy information based on robustness analysis is presented. First, ELECTRE-III is transformed into a continuous smooth function of each parameter vector. Then, robustness analysis structure and a parameters inferring algorithm are provided by maximizing robustness margin based on mathematics programming. Moreover, how to resolve the programming problem is also discussed. At last, a illustrative example of Naval Gun Weapon System Integration is put forward.

Key words. Robustness analysis; Multiple attributes decision making; ELECTRE-III; naval gun weapon system
\end{abstract}

\section{Introduction}

The shipborne weapon system has became more enormous, more complex and more expensive to cope with increasing surface and air threat, which will conflict with relatively skimpy military budget and limited loadage capability. As a subsystem of warship combat system, naval gun weapon system plays an important role and takes on many missions. How to obtain an optimal system integration plan, which will make the efficiency, cost, rate of progress, life force, reliability, maintainability, percent of tonnage to be a suited level generally, is always a pivotal problem for weapon system analysis expert and warship design department. MADM methods have been applied in the integration of many weapon system. Classical parameters (such as weights, cut or threshold) setting methods in MADM methods usually base on decision makers' common sense or intuition, and result is often rigid. Due to human experience's uncertainty, this way is more or less arbitrary which may minify the decision making's creditability. There are two approaches that acknowledge this difficulty and try to remedy it: sensitivity analysis and robustness analysis.

Sensitivity analysis is a way to estimate how much the parameters vary when leading to a constant result, or to find which is the most sensitive parameter and which is a substitute action for the optimal. The studies about sensitivity analysis are numerous: Zuoprovided a sensitivity analysis way for simple additive weighting method (a kind of classical MADM method) ${ }^{[1]}$; Rios Inusu presented a studying framework on sensitivity analysis, which included the concepts of possible optimal alternative, neighboring possible optimal alternative and sensitivity index ${ }^{[2]}$. But sensitivity analysis has its disadvantage of requiring an 'estimated' center value for each parameter and only considering the corresponding solution. It is also often performed on a single parameter at a time, and ignores the possible interdependencies between parameters. Furthermore, sensitivity analysis is theoretic rather than practical.

Robustness analysis is a hotspot in recent years 
which wins academe's great attention, and is supposed to be an effective way replacing sensitivity analysis on processing imperfect information in MADM. Compared to sensitivity analysis, robustness analysis considers parameters combination rather than a single parameter at a time. It tries to know whether the conclusion changes (or not) when the parameter values vary, rather than how the parameters vary without changing conclusions. Moreover, it has a more extensive consideration, including environment parameters, method models and model parameters. There some representative contribution including the studies of Roy, Vincke, Kouvelis and $\mathrm{Yu}$, Dias, etc., which exploited our scope on robustness analysis. Vincke proposed a formalism to define the concepts of robust solution and robust method. His concept of robustness is that no solution contradicts the first one ${ }^{[3-5]}$; Kouvelis and Yudefine robust solution as a rank with the best worst-case behavior in the context of discrete optimization problems ${ }^{[6]}$; Dias defines robust solution as a scenarios combination that holds the rank unchanged with fuzzy information ${ }^{[7]}$, which is also the background concept of this paper. In sum, Robustness analysis is a new direction in MADM, which emphasizes that uncertain factors should be considered, and the optimal solution must be also robust and neutral.

ELECTRE- III is one kind of important MADM methods based on valued outranking relation. How to set parameters is an important step for ELECTRE-III. In the second section, the problem to be discussed is formulized, including ELECTRE- III method, it's transform and the fuzzy information's denotation. In the third section, a frame of robustness analysis method based on classical optimization theory is presented, which is applied in inferring ELECTRE-III's parameters in the fourth section. First, the constraints are transformed into continuous smooth functions of parameters vector. Then an inferring algorithm of parameters is provided by maximizing robustness margin based on mathematics programming. The fifth section provides an illustrative example of naval gun weapon system integration as a case study on the method of inferring ELECTRE- III ' $s$ parameters. Furthermore, it is discussed that this method can also be applied to other MADM methods in the last section. This paper is also an extension of a previous work of the authors. The Ref.[8] only provided a frame work on inferring ELECTRE-III' s parameters and didn't discuss how to solve it. In this paper, it is completed.

\section{Formulation}

\subsection{ELECTRE-III method}

The outranking relation construction is based on the alternatives set $A=\left\{a_{1}, a_{2}, \ldots, a_{n}\right\}$. To obtain the outranking relation between the alternatives $a_{i}$ and $a_{k}$ ( $\left.a_{i}, a_{k} \in A\right)$, concordance test and disconcordance test proceed as follows:

\section{1) Basic information}

Let $J=\{j \in J: j=1,2, \cdots, m\}$ denote the set of attribute indices. $y_{j}\left(a_{i}\right)$ denotes the evaluation of an alternative $a_{i} \in A$ on the attribute $j$. All the attributes are assumed to be benefit type, namely the higher the value, the better it is. Let $w=\left\{w_{1}, w_{2}, \cdots, w_{m}\right\}$ denote the set of weights proposed by decision makers. $q_{j}$ is preference indifference threshold between alternatives on the $j$-th attribute, $p_{j}$ denote strictly preference threshold , and $v_{j}$ denote veto threshold. Namely when the preference attribute value between $y_{j}\left(a_{i}\right)$ and $y_{j}\left(a_{k}\right)$ exceeds $v_{j}$, the judgment that alternative $a_{i}$ generally outranks $a_{k}$ is no longer accepted.

2) Define concordance indices and disconcordance indices

$C\left(a_{i}, a_{k}\right)$ is defined as concordance indices of alternative couples $\left(a_{i}, a_{k}\right) \in A^{2}$ in ELECTRE- III method:

$$
C\left(a_{i}, a_{k}\right)=\sum_{j=1}^{m} w_{j} \cdot c_{j}\left(a_{i}, a_{k}\right) / \sum_{j=1}^{m} w_{j}
$$

where

$$
c_{j}\left(a_{i}, a_{k}\right)= \begin{cases}0 \quad & \text { if } y_{j}\left(a_{i}\right)-y_{j}\left(a_{k}\right) \leq q_{j} \\ 1 \quad & \text { if } y_{j}\left(a_{i}\right)-y_{j}\left(a_{k}\right) \geq p_{j} \\ \frac{y_{j}\left(a_{i}\right)-y_{j}\left(a_{k}\right)-q_{j}}{p_{j}-q_{j}} & \text { otherwise }\end{cases}
$$

Index $c_{j}\left(a_{i}, a_{k}\right)$ denotes the degree of preference $a_{i}$ over $a_{k}$ on the $j$-th attribute, and $C\left(a_{i}, a_{k}\right)$ denotes the measurement that sustains the judgment " $a_{i}$ outrank $a_{k}$ ".

Disconcordance indices is $d_{j}\left(a_{i}, a_{k}\right)$ :

$$
d_{j}\left(a_{i}, a_{k}\right)=\left\{\begin{array}{cc}
0 & \text { if } y_{j}\left(a_{k}\right)-y_{j}\left(a_{i}\right) \leq p_{j} \\
1 \quad & \text { if } y_{j}\left(a_{k}\right)-y_{j}\left(a_{i}\right) \geq v_{j} \\
\frac{y_{j}\left(a_{k}\right)-y_{j}\left(a_{i}\right)-p_{j}}{v_{j}-p_{j}} & \text { otherwise }
\end{array}\right.
$$

$d_{j}\left(a_{i}, a_{k}\right)$ denotes the measurement that rejects the judgment " $a_{i}$ outrank $a_{k}$ ". 


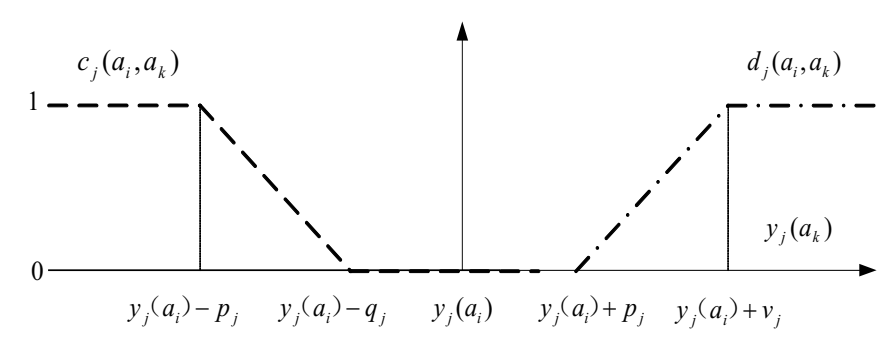

Figure 1. Definition of $c_{j}\left(a_{i}, a_{k}\right)$ and $d_{j}\left(a_{i}, a_{k}\right)$ functions

In Figure 1, horizontal axis denotes $y_{j}\left(a_{k}\right)$, vertical axis denotes the function values of $c_{j}\left(a_{i}, a_{k}\right)$ and $d_{j}\left(a_{i}, a_{k}\right)$, in which broken line is $c_{j}\left(a_{i}, a_{k}\right)$ function, and dashdotted line is $d_{j}\left(a_{i}, a_{k}\right)$ function.

\section{Suppose}

$$
\begin{aligned}
& J^{+}=\left\{j \in J \mid d_{j}\left(a_{i}, a_{k}\right) \leq C\left(a_{i}, a_{k}\right)\right\} \\
& J^{-}=\left\{j \in J \mid d_{j}\left(a_{i}, a_{k}\right)>C\left(a_{i}, a_{k}\right)\right\}
\end{aligned}
$$

$J^{+}\left(a_{i}, a_{k}\right)$ denotes the subset of $J$ that $d_{j}\left(a_{i}, a_{k}\right) \geq C\left(a_{i}, a_{k}\right), J^{-}\left(a_{i}, a_{k}\right)$ denotes the subset that $d_{j}\left(a_{i}, a_{k}\right)<C\left(a_{i}, a_{k}\right)$.

\section{3) Define valued outranking relationship}

Define valued outranking relationship between alternative couple $\left(a_{i}, a_{k}\right)$ as $S\left(a_{i}, a_{k}\right)$ :

$$
S\left(a_{i}, a_{k}\right)= \begin{cases}C\left(a_{i}, a_{k}\right) & \text { if } \forall j \in J^{+} \\ C\left(a_{i}, a_{k}\right) \prod_{j \in J^{-}\left(a_{i}, a_{k}\right)} \frac{1-d_{j}\left(a_{i}, a_{k}\right)}{1-C\left(a_{i}, a_{k}\right)} & \text { if } \forall j \in J^{-}\end{cases}
$$

$S\left(a_{i}, a_{k}\right)$ denotes the measurement that sustains the judgment " $a_{i}$ generally outrank $a_{k}$ ".

Suppose

$$
D_{j}\left(a_{i}, a_{k}\right)= \begin{cases}1 & \text { if } \forall j \in J^{+} \\ \frac{1-d_{j}\left(a_{i}, a_{k}\right)}{1-C\left(a_{i}, a_{k}\right)} & \text { if } \forall j \in J^{-}\end{cases}
$$

Then

$$
D\left(a_{i}, a_{k}\right)=\prod_{j \in J} D_{j}\left(a_{i}, a_{k}\right)
$$

Then

$$
S\left(a_{i}, a_{k}\right)=C\left(a_{i}, a_{k}\right) \cdot D\left(a_{i}, a_{k}\right)
$$

By the valued outranking relation $S\left(a_{i}, a_{k}\right)$, it is possible to define a family of nested crisp outranking relations $S_{\lambda}$ :

$$
S_{\lambda}=\left\{\left(a_{i}, a_{k}\right) \in A \times A: S\left(a_{i}, a_{k}\right) \geq \lambda\right\}, \lambda \in[0.5,1](9)
$$

$\lambda$ is belief level that $S\left(a_{i}, a_{k}\right)$ satisfies $S_{\lambda}$.

\subsection{Transformed ELECTRE-III}

From the formulas $(6) \sim(8)$, there is a nonlinear relationship between $S\left(a_{i}, a_{k}\right)$ and $w$, which is difficult to be solved. Furthermore the subset of attributes $J^{-}$can't be integrated into programming function. The Ref. [9] had studied the weights and veto thresholds in $S\left(a_{i}, a_{k}\right)$, and also considered that $S\left(a_{i}, a_{k}\right)$ is a continuous undifferentiable nonlinear concave function of $w$.

To make classical optimization methods applied easily, $S\left(a_{i}, a_{k}\right)$ is transformed as follows.

Transformed disconcordance index $d^{\prime}{ }_{j}\left(a_{i}, a_{k}\right)$ is :

$$
d^{\prime}{ }_{j}\left(a_{i}, a_{k}\right)=\left\{\begin{array}{cc}
0 & \text { if } y_{j}\left(a_{k}\right)-y_{j}\left(a_{i}\right) \leq u_{j} \\
1 & \text { if } y_{j}\left(a_{k}\right)-y_{j}\left(a_{i}\right) \geq v_{j} \\
\frac{y_{j}\left(a_{k}\right)-y_{j}\left(a_{i}\right)-u_{j}}{v_{j}-u_{j}} & \text { otherwise }
\end{array}\right.
$$

$u_{j}$ is veto indifference threshold, $p_{j} \leq u_{j} \leq v_{j}$.

Transformed $d^{\prime}{ }_{j}\left(a_{i}, a_{k}\right)$ is shown as Figure 2:

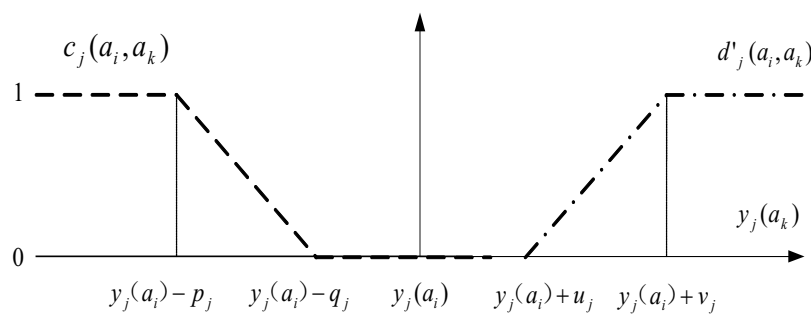

Figure 2. Functions of $c_{j}\left(a_{i}, a_{k}\right)$ and $d^{\prime}{ }_{j}\left(a_{i}, a_{k}\right)$

Transformed $D^{\prime}{ }_{j}\left(a_{i}, a_{k}\right)$ is:

$$
D^{\prime}{ }_{j}\left(a_{i}, a_{k}\right)= \begin{cases}1 & \text { if } \forall j \in J^{+} \\ 1-d^{\prime}{ }_{j}\left(a_{i}, a_{k}\right) & \text { if } \forall j \in J^{-}\end{cases}
$$

Then

$$
\begin{gathered}
D_{j}^{\prime}\left(a_{i}, a_{k}\right)=\operatorname{Min}\left\{1,1-d^{\prime}{ }_{j}\left(a_{i}, a_{k}\right)\right\} \\
D^{\prime}\left(a_{i}, a_{k}\right)=\prod_{j} D^{\prime}{ }_{j}\left(a_{i}, a_{k}\right)
\end{gathered}
$$

The transformed $S\left(a_{i}, a_{k}\right)$ is:

$$
\begin{gathered}
S^{\prime}\left(a_{i}, a_{k}\right)=C\left(a_{i}, a_{k}\right) \cdot D^{\prime}\left(a_{i}, a_{k}\right) \\
S_{\lambda}^{\prime}=\left\{\left(a_{i}, a_{k}\right) \in A \times A: S^{\prime}\left(a_{i}, a_{k}\right) \geq \lambda\right\}
\end{gathered}
$$

\subsection{Fuzzy information's denotation}

Usually, decision maker is difficult to provide exact parameters, but may present some linear constraints easily, by which an fuzzy information space can be constructed. It is formed as follows:

1) Weights space 
(a) $w_{j}^{l} \leq w_{j} \leq w_{j}^{u}$;

(b) $\alpha_{1} w_{1}+\alpha_{2} w_{2}+\ldots+\alpha_{m} w_{m} \geq 0$;

(c) $\beta_{0}+\beta_{1} w_{1}+\beta_{2} w_{2}+\ldots+\beta_{m} w_{m}=0$.

All these constraints can be denoted as the space $W:$

$$
w=\left(w_{1}, w_{2}, \ldots, w_{m}\right) \in W \subset \mathrm{R}^{m}
$$

2) Cutting level space

$$
\lambda^{l} \leq \lambda \leq \lambda^{u}
$$

Denoted as the space $\Lambda$ :

$$
\lambda \in \Lambda \subset \mathrm{R}
$$

3) Preference threshold space

$$
p_{j}^{l} \leq p_{j} \leq p^{u}{ }_{j}, j \in J
$$

Denoted as :

$$
p=\left(p_{1}, p_{2}, \ldots, p_{m}\right) \in P \subset \mathrm{R}^{m}
$$

4) Preference indifference threshold space

$$
q_{j}^{l} \leq q_{j} \leq q_{j}^{u}, j \in J
$$

Denoted as :

$$
q=\left(q_{1}, q_{2}, \ldots, q_{m}\right) \in Q \subset \mathrm{R}^{m} .
$$

5) Veto threshold space

(a) $v_{j}^{l} \leq v_{j} \leq v^{u}{ }_{j}, j \in J$;

(b) $v_{j} \geq p_{j} \geq q_{j}$;

(c) $v_{j}=p_{j}+\beta_{j} \cdot w_{j}$.

Denoted as :

$$
v=\left(v_{1}, v_{2}, \ldots, v_{m}\right) \in V \subset \mathrm{R}^{m}
$$

6) Veto indifference threshold space
(a) $u_{j}^{l} \leq u_{j} \leq u_{j}^{u}, \quad j \in J$
(b) $v_{j} \geq u_{j} \geq p_{j}$;
(c) $u_{j}=p_{j}+\gamma_{j} \cdot\left(v_{j}-p_{j}\right)$.

Denoted as :

$$
u=\left(u_{1}, u_{2}, \ldots, u_{m}\right) \in U \subset \mathrm{R}^{m}
$$

Suppose

$$
\delta=\{w, \lambda, p, q, v, u\}
$$

The parameters space $\Delta$

$$
\Delta=W \otimes \Lambda \otimes P \otimes Q \otimes V \otimes U
$$

Then

$$
\delta \in \Delta
$$

\section{A frame of robustness analysis}

A formalized definition of robustness is provided in the Ref. [10]:

Definition 1: A conclusion $C^{r}$, is said to be robust with respect to a domain $\Delta$, of possible values for the preference and technical parameters, if there is no a particular set of parameters, $\delta \in \Delta$, which invalidates the conclusion $C^{r}$.

The problem of inferring parameters in ELECTREIII is solved by maximizing robustness margin based on mathematics programming.

Let

$$
\begin{aligned}
& S^{+}=\left\{\left(a_{i}, a_{k}\right) \mid S^{\prime}\left(a_{i}, a_{k}\right) \geq \lambda\right\} \\
& S^{-}=\left\{\left(a_{i}, a_{k}\right) \mid S^{\prime}\left(a_{i}, a_{k}\right)<\lambda+\varepsilon\right\}
\end{aligned}
$$

$\varepsilon$ is a tiny positive number.

To keep the rank constant, the set $S^{+}$and $S^{-}$ must be unchanged. So, the optimal parameters vectors can also be got by maximizing robustness margin with the constraint conditions $S^{+}$and $S^{-}$.

$$
\begin{cases}\operatorname{Max} & \alpha(\delta) \\ \text { s.t. } & \alpha \leq S\left(a_{i}, a_{k}\right)-\lambda \forall\left(a_{i}, a_{k}\right) \in S^{+} \\ & \alpha \leq \lambda-S\left(a_{i}, a_{k}\right)+\varepsilon \forall\left(a_{i}, a_{k}\right) \in S^{-} \\ & \delta \in \Delta, j \in J\end{cases}
$$

$\delta$ denotes every parameters in the section 2.3 , and $\Delta$ denotes constrained domain.

Definition 2: Let

$$
\alpha=S^{\prime}\left(a_{i}, a_{k}\right)-\lambda
$$

$\alpha$ is Robustness Margin of alternative $a_{i}$ outranking $a_{k}, 0 \leq \alpha \leq 1$.

The rank is more robust when $\alpha$ is larger.

Definition 3: If the programming (17) have solution, the maximum of $\alpha\left(\alpha_{\max }\right)$ can be obtained which is called 'maximum robustness margin', and the solution is 'optimal parameter vector' $\delta_{\text {opt }}$.

ELECTRE-III's parameters inferring algorithm:

1) Problem formulation: Define alternatives set $A$ and attributes set $J$; Experts assign evaluation values $y_{j}\left(a_{i}\right)$.

2) Set initial parameters : Set initial parameters $\delta_{0}$, parameters constraints, and other ELECTRE- III 's parameters;

3) $S^{+}$and $S^{-}$: Obtain $S^{+}$and $S^{-}$by ELECTRE-III;

4) $\alpha_{\max }$ and $\delta_{\text {opt }}$ : Solve the programming (17) to obtain maximum robustness margin $\alpha_{\max }$ and optimal parameter vector $\delta_{\text {opt }}$;

5) $S^{\prime}\left(a_{i}, a_{k}\right)$ : Obtain the indices $S^{\prime}\left(a_{i}, a_{k}\right)$ by ELECTRE-III with $\delta_{\text {opt }}, \forall a_{i}, a_{k} \in A$;

6) Rank: Obtain the rank of set $A$ by $S^{\prime}\left(a_{i}, a_{k}\right)$.

\section{Inferring ELECTRE-III's parameters}


The parameters structure of ELECTRE-III is listed in Figure 3. The parameters to be inferred based on robustness analysis include local parameters and global parameters. When local parameters are studied, the others are fixed. In this paper, weights, cutting level, thresholds and other local parameters will be studied respectively. (The parameters to be discussed are at the left half of the Figure 3.) It is more difficult to infer global parameters, which will be discussed in later papers.

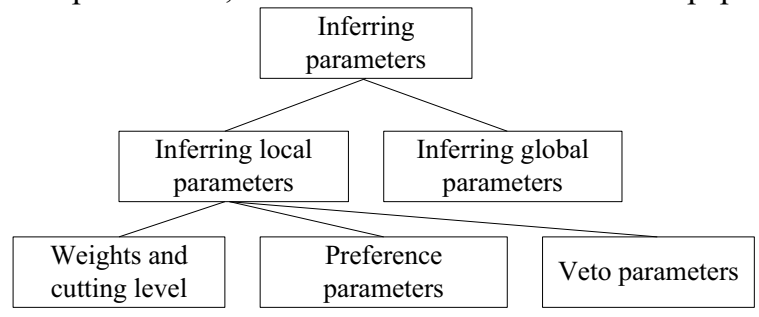

Figure. 3. Parameters structure to be Inferred

\subsection{Inferring Weights and cutting level}

When the parameters weights and the cutting level are analyzed, others parameters are fixed. The constraints of (17) can be embodied as follow:

$$
\begin{cases}\text { Max } & \alpha(w, \lambda) \\ \text { s.t. } & \alpha \leq \sum_{j=1}^{m} w_{j} \cdot c_{j}\left(a_{i}, a_{k}\right) \cdot D^{\prime}\left(a_{i}, a_{k}\right) / \sum_{j=1}^{m} w_{j}-\lambda \forall\left(a_{i}, a_{k}\right) \in S^{+} \\ & \alpha \leq \lambda-\sum_{j=1}^{m} w_{j} \cdot c_{j}\left(a_{i}, a_{k}\right) \cdot D^{\prime}\left(a_{i}, a_{k}\right) / \sum_{j=1}^{m} w_{j}+\varepsilon \forall\left(a_{i}, a_{k}\right) \in S^{-} \\ & \sum_{j=1}^{m} w_{j}=1, w_{j} \geq \varepsilon, w \in W, \forall j \in J, \lambda \in \Lambda\end{cases}
$$

The programming (18) is a linear programming of weights $w$ and cutting level $\lambda$. The optimal estimation of $w$ and $\lambda$ can be obtained by simplex method.

\subsection{Inferring Preference parameters}

1) Inferring preference threshold $q_{j}$ and preference indifference threshold $p_{j}$

When inferring preference threshold $q_{j}$ and preference indifference threshold $p_{j}$ at single attribute $j$, other parameters are fixed. The programming (17) is segmented and concave is difficult to solved. A eclectic method is to smooth the segmented function $c_{j}\left(a_{i}, a_{k}\right)$ :

$$
c_{j}\left(a_{i}, a_{k}\right)= \begin{cases}0 & \text { if } \Delta_{j}\left(a_{i}, a_{k}\right) \leq q^{u}{ }_{j} \\ 1 & \text { if } \Delta_{j}\left(a_{i}, a_{k}\right) \geq p^{l}{ }_{j} \\ \left(\Delta_{j}\left(a_{i}, a_{k}\right)-q_{j}\right) /\left(p_{j}-q_{j}\right) & \text { otherwise }\end{cases}
$$
follow:

$$
\begin{cases}\operatorname{Max} & \alpha\left(p_{j}, q_{j}\right) \\ \text { s.t. } & \left(\frac{\alpha+\lambda}{D^{\prime}}-C_{-j}\right) \cdot \sum_{j=1}^{m} w_{j} / w_{j} \leq c_{j}\left(a_{i}, a_{k}\right) \forall\left(a_{i}, a_{k}\right) \in S^{+} \\ & -\left(\frac{\alpha+\lambda+\varepsilon}{D^{\prime}}-C_{-j}\right) \cdot \sum_{j=1}^{m} w_{j} / w_{j} \leq-c_{j}\left(a_{i}, a_{k}\right) \forall\left(a_{i}, a_{k}\right) \in S^{-} \\ & p^{l}{ }_{j} \leq p_{j} \leq p^{u}{ }_{j}, q^{l}{ }_{j} \leq q_{j} \leq q^{u}{ }_{j}, j \in J \\ & C_{-j}\left(a_{i}, a_{k}\right)=C\left(a_{i}, a_{k}\right)-w_{j} \cdot c_{j}\left(a_{i}, a_{k}\right) / \sum_{j=1}^{m} w_{j} 。\end{cases}
$$

If the programming (20) has solution, the optimal preference threshold $p_{j}^{\text {opt }}$ and optimal preference indifference threshold vector $q_{j}^{\text {opt }}$ can be obtained.

2) Inferring preference threshold vector $q$ and preference indifference threshold vector $p$

The segmented function $c_{j}\left(a_{i}, a_{k}\right)$ is smoothed in the same way as above. If the programming problem has solution, the optimal preference threshold vector $p_{\text {opt }}$ and optimal preference indifference threshold vector $q_{\text {opt }}$ can be obtained.

\subsection{Inferring Veto parameters}

1) Inferring veto threshold $v_{j}$ and veto indifference threshold $u_{j}$

Other parameters are fixed when veto threshold $v_{j}$ and veto indifference threshold $u_{j}$ of single attribute $j$ of single attribute $j$ are inferred. The programming (17) is also is segmented and concave. The smoothed function $d^{\prime}{ }_{j}\left(a_{i}, a_{k}\right)$ is:

$$
d^{\prime}{ }_{j}\left(a_{i}, a_{k}\right)=\left\{\begin{array}{cc}
0 & \text { if } \Delta_{j}\left(a_{k}, a_{i}\right) \leq u_{j}^{l} \\
1 & \text { if } \Delta_{j}\left(a_{k}, a_{i}\right) \geq v^{u}{ }_{j} \\
\left(\Delta_{j}\left(a_{i}, a_{k}\right)-u_{j}\right) /\left(v_{j}-u_{j}\right) \quad \text { otherwise }
\end{array}\right.
$$

Then:

$$
\begin{cases}\operatorname{Max} \alpha\left(u_{j}, v_{j}\right) \\ \text { s.t. } \quad(\alpha+\lambda) / C \cdot D_{-j}{ }^{\prime} \leq d^{\prime}{ }_{j}\left(a_{i}, a_{k}\right) \forall\left(a_{i}, a_{k}\right) \in S^{+} \\ \quad-(\alpha+\lambda+\varepsilon) / C \cdot D_{-j}{ }^{\prime} \leq-d^{\prime}{ }_{j}\left(a_{i}, a_{k}\right) \forall\left(a_{i}, a_{k}\right) \in S^{-} \\ \quad u_{j}{ }_{j} \leq u_{j} \leq u_{j}{ }_{j}, v^{l}{ }_{j} \leq v_{j} \leq v^{u}{ }_{j}, j \in J \\ D_{-j}{ }^{\prime}=D^{\prime} / d^{\prime}{ }_{j}\left(a_{i}, a_{k}\right) \circ\end{cases}
$$

If the programming MP3 has solution, the optimal veto threshold $v_{j}^{\text {opt }}$ and optimal veto indifference threshold vector $u_{j}^{\text {opt }}$ can be obtained.

2) Inferring veto threshold vector $q$ and veto indifference threshold vector $p$ 
The segmented function $d^{\prime}{ }_{j}\left(a_{i}, a_{k}\right)$ is smoothed in the same way as above. If the programming problem has solution, the optimal veto threshold vector $v_{\text {opt }}$ and optimal veto indifference threshold vector $u_{\text {opt }}$ can be obtained.

\section{A case study of naval gun weapon system integration}

As the first step of the process to obtain an optimal integration plan of naval gun weapon system, it is to design some systems that satisfy basis sea battle need and other constrain conditions, namely feasible integration alternatives. Classical system structure of feasible integration alternatives is expressed as below:

(Main sensor) $\cap$ (Spare sensor) + ( Director $)+($ Naval gun firepower system)

Main sensor is track radar; Spare sensor is photoelectric device; Director includes some fire control equipments; Naval gun firepower system include some types of naval guns such as AK630, 37F, AK76 or their combination. Three feasible configuration alternatives on certain type warship are listed as Table 1.

Table 1. Three Feasible Integration Alternatives

\begin{tabular}{ccccc}
\hline & Sensor & $\begin{array}{c}\text { Spare } \\
\text { Sensor }\end{array}$ & Director & $\begin{array}{c}\text { Naval gun firepower } \\
\text { system }\end{array}$ \\
\hline$a_{1}$ & 344 radar & GD-2 & ZPJ-2B & $1 \times$ AK76 \\
& & & & $1 \times \mathrm{AK} 630$ \\
$a_{2}$ & $347 \mathrm{G}$ radar & JM-83 & ZPJ-2B & $3 \times 37 \mathrm{~F}$ \\
$a_{3}$ & TR47 radar & OFD-b30 & CC24 & $2 \times 37 \mathrm{~F} 1 \times$ AK76 \\
\hline
\end{tabular}

Decision makers attach different regard on every attribute in terms of special integration problem. Weights reflect decision maker's preference. Suppose attributes set of naval gun weapon system is $w$,

$w=\{$ efficiency; cost; risk; applicability; compatibility; life force $\}=\left\{y_{1}, y_{2}, y_{3}, y_{4}, y_{5}, y_{6}\right\}$

According to the algorithm of section 3 , the process of naval gun weapon system integration is listed as bellow:

1) Standardized attributes values of three feasible integration alternatives are provided in Table 2, initial weights vector $w_{0}$ is listed in Tab. 3. Parameters constraints are set as follows, which are more or less simple but has it's generality:

$$
\begin{aligned}
& \quad 0.3 \leq w_{1} \leq 0.5 \quad, \quad 0.1 \leq w_{2} \leq 0.2 \quad, \quad w_{1} \geq 2 w_{2}, \\
& 0.1 \leq w_{3} \leq 0.2 \quad, \quad 0.1 \leq w_{4} \leq 0.2 \quad, \quad \varepsilon \leq w_{5} \leq 0.1 \\
& 0.1 \leq w_{6} \leq 0.2, \varepsilon=0.001,0.4 \leq \lambda \leq 0.6,0.5 \leq m_{j} \leq 0.7, \\
& 0.5 \leq p_{j} \leq 0.7,0 \leq q_{j} \leq 0.2,0.5 \leq \sigma_{j} \leq 0.7 .
\end{aligned}
$$

Table 2. Standardized Attributes Values

\begin{tabular}{ccccccc}
\hline & $y_{1}$ & $y_{2}$ & $y_{3}$ & $y_{4}$ & $y_{5}$ & $y_{6}$ \\
\hline$a_{1}$ & 0.4103 & 0.3529 & 0.1389 & 0.6038 & 0.0153 & 0.9318 \\
$a_{2}$ & 0.8936 & 0.8132 & 0.2028 & 0.2722 & 0.7468 & 0.4660 \\
$a_{3}$ & 0.0579 & 0.0099 & 0.1987 & 0.1988 & 0.4451 & 0.4186 \\
\hline
\end{tabular}

2) The result of ELECTRE- III with initial parameters:

$$
a_{2} \succ a_{1}, a_{2} \succ a_{3}
$$

3) Resolve programming (18). The optimal weights vector $w_{\mathrm{opt}}$ are listed in Table $3, \lambda_{\mathrm{opt}}=0.4$.

Table 3. Initial and optimal weights vector $w_{\mathrm{opt}}$

\begin{tabular}{ccccccc}
\hline & $w_{1}$ & $w_{2}$ & $w_{3}$ & $w_{4}$ & $w_{5}$ & $w_{6}$ \\
\hline$w_{0}$ & 0.4 & 0.14 & 0.1 & 0.13 & 0.08 & 0.15 \\
$w_{\text {opt }}$ & 0.40 & 0.15 & 0.10 & 0.20 & 0.001 & 0.149 \\
\hline
\end{tabular}

4) Resolve programming (20) and (22). The optimal threshold vectors are listed in Table 4 :

Table 4. The optimal threshold vectors

\begin{tabular}{lcccccc}
\hline Attribute & 1 & 2 & 3 & 4 & 5 & 6 \\
\hline$\left.p_{\text {opt }}, q_{\text {opt }}\right)$ & $(0.7,02)$ & $(0.5,0.2)$ & $(0.5,0.2)$ & $(0.6,0.1)$ & $(0.7,02)$ & $(0.7,0.2)$ \\
$\left(u_{\text {opt }}, v_{\text {opt }}\right)$ & $(0.7,02)$ & $(0.6,0.1)$ & $(0.6,0.1)$ & $(0.6,0.1)$ & $(0.7,0.2)$ & $(0.7,02)$ \\
\hline
\end{tabular}

5) The final result of ELECTRE-III with optimal parameters:

$$
a_{2} \succ a_{3} \succ a_{1}
$$

\section{Summary and conclusions}

In this paper, a robustness analysis method is presented in terms of inferring ELECTRE- III 's parameters. The programming (18) is linear, convex, and can be solved by simplex method easily; but the programming (20) and (22) are nonlinear, concave and difficult to be solved, which need further study to find more proper way.

On the other hand, this method can also be applied to infer parameters in other MADM methods. For example, for AHP method or TOPSIS method, which are based on the utility theory, the $S\left(a_{i}, a_{k}\right)$ can be defined as :

$$
S\left(a_{i}, a_{k}\right)=\phi\left(a_{i}\right)-\phi\left(a_{k}\right)
$$

$\phi\left(a_{i}\right), \phi\left(a_{k}\right)$ is the evaluation of alternative $a_{i}, a_{k}$ respectively. The optimal parameters can also be obtained by solving the programming (17). 


\section{Acknowledgment}

The author is very grateful to Doctor Xie Jun for his insightful and constructive comments and suggestions that led to an improved version of this article.

\section{References}

[1] J. Zuo, The Discussion on Sensitivity Analysis in Methods Multiple Criteria Decision Making, System Engineering Theory and Application, 1987, 7(3): 1-11.

[2] R. Inusu, S. French. A Framework for Sensitivity Analysis in Discrete Multi-objective Decision-making, European Journal of Operational Research, 1991, 54(1): 176-190..

[3] B. Roy, A Missing Link in OR-DA: Robustness Analysis, Foundations of Computing and Decision Sciences, 1998, 23(1): 141-160.

[4] Ph. Vincke, Robust Solutions And Methods in Decision Aid, Journal of Multi-Criteria Decision Analysis 1999, 8(1):181-187.

[5] Ph. Vincke, Robust and Neutral Methods for Aggregating Preferences into an Outranking Relation, European Journal of Operational Research, 1999, 112(2): 405-412.

[6] Ph. Vincke, About the Application of MCDM to Some Robustness Problems, European Journal of Operational Research, 2006. 176(3): 645-658.

[7] P. Kouvelis, G. Yu, Robust Discrete Optimization and Its Application, New York: Kluwer Academic Publishers, 1997.

[8] S.Y.,Sun, Z.M.,Qiu, H.Y.,Wang ELECTRE-III's Parameters Inferring Method Based on Robustness Analysis, Command Control \& Simulation, 2006, 28(6):14-18.

[9] L. C. Dias, J. N. Climaco, On Computing ELECTRE's Credibility Indices under Partial Information , Journal of Multi-Criteria Decision Analysis 1999, 8(1):74-92.

[10]J. Figueira(Ed.), Multiple Criteria Decision Analysis: State of the Art Surveys, New York: Kluwer Academic Publishers, 2005: 346 